\title{
Resting-state networks in adolescents with 22q11.2 deletion syndrome: Associations with prodromal symptoms and executive functions
}

\author{
Martin Debbané a,b,*, Marine Lazouret ${ }^{\mathrm{a}, \mathrm{b}}$, AnnaLaura Lagioia a , Maude Schneider ${ }^{\mathrm{a}}$, \\ Dimitri Van De Ville ${ }^{\mathrm{c}, \mathrm{d}}$, Stephan Eliez ${ }^{\mathrm{a}, \mathrm{e}}$
}

\footnotetext{
a Office Médico-Pédagogique Research Unit, Department of Psychiatry, University of Geneva School of Medicine, Switzerland

b Adolescent Clinical Psychology Research Unit, Faculty of Psychology, University of Geneva, Switzerland

' Department of Radiology and Medical Informatics, University of Geneva, Switzerland

d Institute of Bioengineering, Ecole Polytechnique Fédérale de Lausanne, Lausanne, Switzerland

e Department of Genetic Medicine and Development, University of Geneva School of Medicine, Switzerland
}

\section{A R T I C L E I N F O}

\section{Article history:}

Received 8 February 2012

Received in revised form 30 April 2012

Accepted 24 May 2012

Available online 14 June 2012

\section{Keywords:}

Velo-cardio-facial syndrome (VCFS)

Psychosis

Schizophrenia

Default-mode network

Attention

Executive functions

\begin{abstract}
A B S T R A C T
Atypical functional connectivity in the maturing brains of 22q11.2 deletion syndrome (22q11DS) may contribute to the expression of early psychotic symptoms commonly reported by these youths. This study's objective was to examine functional connectivity in cerebral networks at rest (Resting-State Networks; RSNs) and their relationship to symptomatic and neuropsychological characteristics putting them at very high risk factor for developing psychosis. Twenty-seven adolescents with 22q11DS and 33 typically developing control adolescents matched for age, gender and handedness underwent an 8-minute resting state functional MRI session. RSNs identification procedure employed Independent Component Analysis (ICA). We tested for potential group differences in functional connectivity within-networks. Then, we examined relationships between network connectivity and symptomatic/neuropsychological characteristics in the 22q11DS group. A total of nine resting-state networks were identified. Between-group differences suggested both increased and decreased functional connectivity in the 22q11DS group, involving the default-mode, sensorimotor, visuo-spatial, and high level visual networks. Finally, atypical connectivity in the default-mode network, specifically within the left superior frontal gyrus region, correlated with prodromal symptom intensity and neuropsychological performances in the 22q11DS group. The results suggest that atypical functional connectivity may sustain both increased vulnerability to psychosis and characteristic cognitive impairments in 22q11DS.
\end{abstract}

(c) 2012 Elsevier B.V. All rights reserved.

\section{Introduction}

A growing concern for adolescents with 22q11.2 deletion syndrome (22q11DS) is fuelled by self-report evaluations and interview-based assessments suggesting that $45 \%$ experience transient psychotic symptom of moderate to severe intensity (Stoddard et al., 2010). 22q11DS is most commonly described as a congenital disorder caused by a $3 \mathrm{Mb}$ microdeletion on the long arm of chromosome 22, affecting 1/4300-7000 live births (Oskarsdottir et al., 2004). Cognitive delay, as well as increased prevalence of attention deficits, mood and anxiety disorders, is characteristic of this population (De Smedt et al., 2007a; Green et al., 2009). Concern relating to psychological health in these adolescents is matched by the neuroscientists' efforts to identify the neural markers associated with early schizotypal trait expression in 22q11DS (Schaer et al., 2009a; Gothelf et al., 2011). Indeed, in this deletion

\footnotetext{
* Corresponding author at: Adolescent Clinical Psychology Research Unit, Faculty of Psychology, University of Geneva, Switzerland, Boulevard du Pont d'Arve 40, 1204 Geneva, Switzerland. Tel.: +4122379 9418 .

E-mail address: martin.debbane@unige.ch (M. Debbané).
}

syndrome where $25-30 \%$ are thought to evolve into formal schizophrenia spectrum disorders by adulthood (Murphy et al., 1999), neurodevelopment during adolescence may provide key insights to understanding the unfolding of psychotic symptoms.

To date, neuroimaging studies have mainly focused on the structural determinants of cerebral development in 22q11DS, and reports typically highlight deviant maturational courses as characterized by local reductions in brain volume, gray matter density, white matter density, gyrification and cortical thickness (Eisenberg et al., 2010). No study to our knowledge has yet to examine the baseline neural processes that characterize the architecture of functional connectivity in youths with 22q11DS. Such studies may indeed be important, especially because recent investigations in other pediatric populations show that atypical functional connectivity contributes to the emergence of neurodevelopmental psychiatric disorders (Assaf et al., 2010; Fair et al., 2010).

Neurodevelopmental and neurocognitive theories specifically devoted to understanding schizophrenia identify cerebral dysconnectivity as a critical factor in the expression of psychotic symptoms (Friston and Frith, 1995; Hoffman and McGlashan, 2001). Starting from the perspective of 
the healthy brain, cerebral connectivity is functionally organized into distinct neuronal networks able to dynamically sustain different domains of information processing (Raichle and Snyder, 2007). Functional connectivity, defined as the co-activation of spatially distributed areas in the brain, stands for the cooperation and integration of specialized neuronal systems: reduced connectivity within these networks may result in dysfunctional specialization (Friston, 1998). Functional neuroimaging studies examining resting state networks (RSN) in typical adults reveal the organization of the brain's spontaneous activity (mind wandering), which typically encompasses functions such as vision, audition, attention, language, sensorimotor processing, self-referential processing and a default-mode (DMN) (Mantini et al., 2007; Rosazza and Minati, 2011). In essence, estimates of functional connectivity during the brain's resting-state have revealed important information on its basic functional architecture.

In contrast to healthy adults, individuals with schizophrenia exhibit abnormal patterns of resting-state cerebral connectivity (Broyd et al., 2009). To date the findings are inconsistent across studies, reporting both increased and decreased functional connectivity in the DMN of patients with schizophrenia (Greicius, 2008; Broyd et al., 2009). Nevertheless, recent studies seem to converge on characterizing functional connectivity in schizophrenia as generally decreased in modularity and efficiency (Lynall et al., 2010; AlexanderBloch et al., 2012). In the past two years, a number of studies have examined RSNs in populations with specific symptom profiles such as ultra high-risk for schizophrenia (Shim et al., 2010) and childhood onset-schizophrenia (Alexander-Bloch et al., 2010). Accumulating rapidly, these studies are demonstrating how functional connectivity is implicated in the development of psychopathology, sometimes before the unfolding of clinically relevant symptoms that command a need for care (Lagioia et al., 2010). To the best of our knowledge, RSN functional connectivity in youths with 22q11DS, who are at genetic high-risk for developing schizophrenia, still remains to be examined.

This study carries three aims. First, we wish to characterize the resting-state networks (RSNs) in a group of youths affected by the 22q11DS. By using group-level Independent Component Analysis (ICA), which extracts components consisting of spatially distributed networks between temporally coherent brain regions, we employ a data driven approach which is best suited for the first identification of the main RSNs in 22q11DS. Second, we perform group comparisons between the spatial maps to uncover potential differences in RSNs identified by ICA between the 22q11DS group and gender and agematched controls. Finally, based on recent studies suggesting the association of cerebral dysconnectivity in the medial DMN with symptoms of psychosis (Shim et al., 2010; Sorg et al., in press), we examine which potential group differences in this network significantly relate to expression of psychosis. Additionally, we perform post-hoc exploratory correlations with schizophrenia-relevant executive function measures in adolescent participants with 22q11DS.

\section{Materials and methods}

\subsection{Participants}

Twenty-seven adolescents diagnosed with the 22q11DS (12 males) aged between 12 and 19 ( mean $=15.76 \pm 2.09)$ participated in the study. At the time of participation, eight participants with 22q11DS were receiving psychotropic medication at the time of testing: four were on methylphenidate, two on antidepressant (sertaline), one on mood stabilizer (lamotrigine), and two on antipsychotic medication (cyamemazine and risperidone). Participants were recruited through Swiss and European patient associations. Written informed consent was received from all parents and/or subjects under protocols approved by the Institutional Review Board of the Geneva University School of Medicine. The comparison group consisted of thirty-three healthy participants (19 males) in the same age range as the 22q11 DS population (mean: $15.40 \pm 1.94$ ), screened for the absence of neurological and psychiatric disorders through a medical intake interview and screening forms.

Two neuropsychological tasks evaluating executive control and attention were administered. First, the Stroop task was administered to evaluate the interference effect on directed attention. Second, the Conners' Continuous Performance Test II computerized assessment was administered to measure three neuropsychological indices (Omissions, Commissions, Hits).

A child and adolescent psychiatrist used the Diagnostic Interview for Children and Adolescents (DICA) to interview participants with 22q11DS younger than 18 years and their parents. Participants aged 18 years and older were assessed using the Structured Clinical Interview for DSM Disorders (SCID). Participants with 22q11DS underwent the Structured Interview of Prodromal Symptoms (SIPS) (McGlashan, 2001) to assess the full breath of prodromal signs of psychosis in this population (Debbané et al., 2008; Stoddard et al., 2010; Schneider et al., 2012). We followed the Criteria for Prodromal Syndromes (COPS 3.0) in identifying prodromal syndromes. A total of 6 participants with 22q11DS met prodromal criteria, comprising attenuated positive symptoms syndrome $(n=6$; one with comorbid genetic risk and deterioration prodromal syndrome). Additionally, one participant was diagnosed with a psychotic disorder. Descriptive data summarizing the clinical and cognitive characteristics of participants can be found in Table 1 .

\section{2. fMRI data acquisition}

Scanning was performed using a 3 Tesla Trio Tim Siemens with a 16-channel receiver head coil at the Center for Biomedical Imaging in Geneva, Switzerland. High resolution 3D anatomical images were acquired (repetition time $[\mathrm{TR}]=2500 \mathrm{~ms}$, echo time $[\mathrm{TE}]=30 \mathrm{~ms}$, 192 coronal slices, slice thickness $=1.1 \mathrm{~mm}$, no gap, flip angle $=8^{\circ}$, matrix size $224 \times 256$, field of view [FOV] $=256 \times 224$ ), followed by a resting state functional scan of $8 \mathrm{~min}$ (staring at a fixation cross on the screen), containing 200 blood oxygenated level-dependent (BOLD) images (repetition time $[\mathrm{TR}]=2400 \mathrm{~ms}$, echo time $[\mathrm{TE}]=$ $30 \mathrm{~ms}, 38$ axial slices, slice thickness $=3.20 \mathrm{~mm}$, spacing between

\section{Table 1}

Demographics and descriptive data for the 22q11DS and control groups. Mean (sd) is provided in the table unless otherwise specified. Range is also provided for the SIPS scores.

\begin{tabular}{lll}
\hline & 22q11DS & Controls \\
\hline $\begin{array}{l}\text { Demographics } \\
\text { Age }\end{array}$ & $15.76(2.08)$ & $15.46(1.91)$ \\
Gender (\% females) & $15(55.6 \%)$ & $14(42.4 \%)$ \\
SIPS & & \\
Total score & $25.19(14.49) ; 5-69$ & $\mathrm{~N} / \mathrm{A}$ \\
Positive domain & $5.35(5.39) ; 0-23$ & $\mathrm{~N} / \mathrm{A}$ \\
Negative domain & $12.38(6.09) ; 3-30$ & $\mathrm{~N} / \mathrm{A}$ \\
Disorganization domain & $3.84(2.78) ; 0-12$ & $\mathrm{~N} / \mathrm{A}$ \\
General domain & $3.62(4.02) ; 0-14$ & $\mathrm{~N} / \mathrm{A}$ \\
& & \\
WISC/WAIS & $70.70(11.60)$ & $109.11(12.73)(\mathrm{N}=27)^{\mathrm{a}}$ \\
Full-scale IQ & & \\
& & \\
Stroop & $2.61(7.51)$ & $2.42(5.24)$ \\
Interference score & & $50.04(7.20)$ \\
CPT & $55.14(12.62)$ & $39.42(7.00)$ \\
Omission t-score & $57.05(12.89)$ & \\
Commission t-score & $41.50(11.52)$ & \\
Hit RT t-score & & \\
\hline
\end{tabular}

\footnotetext{
a Group difference $p<0.001 ; 6$ control participants underwent a short IQ version that does not allow computing a full-scale IQ score. We reported the IQ scores for the 27 remaining participants in the table.
} 
slices $=3.2 \mathrm{~mm}$, flip angle $=85^{\circ}$, matrix size $94 \times 128$, field of view $[\mathrm{FOV}]=96 \times 128)$. Subject's head was stabilized with a vacuum cushion to minimize motion.

\section{3. fMRI data analysis}

Data were processed and analyzed using Statistical Parametric Mapping (SPM8; Wellcome Trust Centre for Neuroimaging, London, UK: http://www.fil.ion.ucl.ac.uk/spm/). Functional images were at first corrected for motion by realigning each image with respect to the first one. Slice time correction was conducted with the use of the middle slice as reference. Image slices were acquired in ascending order. T1-weighted anatomical image of each individual was then coregistered to the mean realigned functional images. Segmentation of the structural image was run to obtain gray and white matter separation. Realigned and slice-timed images were spatially normalized into the Montreal Neurological Institute (MNI) template using $3 \mathrm{~mm}$ cube isotropic voxels, followed by spatial smoothing with an isotropic Gaussian smoothing Kernel of $8 \mathrm{~mm}$ full width half maximum (FWHM). To control for movement artefacts, subjects with a movement superior to $3 \mathrm{~mm}$ for translation and $3^{\circ}$ for rotation were taken out of the study (Controls $=6,22 q 11 D S=9$ ). To further control for any significant group differences in head movement, we averaged each type of movement for the two groups and compared them between groups. We found no significant differences in translational and rotational movement $(p>0.05)$ between groups.

\subsection{Network identification through independent component analysis (ICA)}

Group-level spatial ICA was conducted on the entire sample of participants using the GIFT implementation (available at http:// icatb.sourceforge.net/) that relies on the InfoMax algorithm. Specifically, spatial ICA allows studying the spatio-temporal structure of the functional data by decomposing into different components that are optimized according to a criterion of spatial independence. The component maps are typically sparsely distributed spatial patterns of temporally coherent brain regions. The spatially pre-processed images of patients and controls were analyzed jointly using group-level analysis with ICASSO ran a hundred times to ensure stability of the ICs. The number of components determined was $n=20$, which is consistent with the minimum description length estimate ( $\mathrm{Li}$ et al., 2007). Each of the spatial components was manually inspected for the presence of obvious artifacts that get separated out by ICA (e.g., motion, ventricles) (Stevens et al., 2007).

\subsection{Group differences for RSN functional connectivity and post-hoc correlation analyses}

Peak activations (t-values) output ICA files for each subject, for all components of interest, were used to run two-sample t-tests in SPM8. We tested for spatial differences in RSNs between 22q11DS patients and controls with a threshold at $p<0.001$ uncorrected. Cluster-level peak functional activity was then estimated each component of interest $(p<0.05$ FWE-corrected).

To examine whether medial DMN group differences were related to psychotic symptoms and neuropsychological characteristics in the 22q11DS group, we extracted brain activity from the medial regions of interest (ROIs) at the cluster level with the SPM8 toolbox EasyROI (available at: http://www.sbirc.ed.ac.uk/LCL/LCL_M1.html). We performed regression analyses using Total SIPS Positive and Negative subscales as dependent variables, and medial DMN ROI values as independent variables. Post-hoc Pearson correlations with individual SIPS symptoms scale values, Stroop interference score and CPT t-scores for total Hits, omission and commission errors finalized our analyses.

\section{Results}

\subsection{ICA identification of resting state networks (RSNs)}

Nine significant RSNs were identified through ICA for the entire group (22q11.2 deletion syndrome (22q11DS) and healthy control (CTRL) participants), retained and designated based on functional terms following Laird et al. (2011) (Fig. 1).

\subsection{Inter-group differences on RSNs}

Group comparisons for functional connectivity within the RSNs yielded significant group differences for the following networks: highlevel visual network (Control $>22 q 11 D S$ ), sensorimotor network (22q11DS $>$ Control), visuo-spatial processing network (22q11DS > Control), and default-mode network (Control > 22q11DS; 22q11DS> Control; Fig. 2a). The same networks are found when we exclude the 7 participants with prodromal syndromes in the 22q11DS group. Table 1 presents peak activation coordinates for significant clusters, together with $\mathrm{T}$ values and corrected $\mathrm{p}$ values.

\subsection{Correlations with psychotic symptom ratings and neuropsychological characteristics}

We extracted beta values for those group differences for which peak cluster activity, within the DMN, was found within significantly different medial activations in our group contrasts. This resulted in the selection of 5 ROIs (see * in Table 2 ). We first performed regression analyses to identify which of these regions might specifically be associated with 1) positive prodromal symptoms; 2) negative prodromal symptoms. The first analysis found that the left superior frontal gyrus (ISFG) ROI $(\beta=-.425, p=.031)$ significantly contributed to the model $\left(\mathrm{R}^{2}=.18, \mathrm{~F}(1,24)=5.28, p=.031\right)$. The ISFG also contributed $(\beta=-.431, p=.028)$ to the regression model for SIPS total negative symptoms' score $\left(\mathrm{R}^{2}=.19, \mathrm{~F}(1,24)=5.47, p=.028\right)$. Post-hoc Pearson's correlations suggested that these results were driven by the 3 subscale score of the SIPS total Positive score (P1: $r=-0.42$, $p=0.035$; P2: $r=-0.54, p=0.004$; P5: $r=-0.53, p=0.005)$ and 2 subscale scores for SIPS total Negative score (N1: $r=-0.47$, $p=0.016$; N3: $r=-0.50, p=0.009)$. After Bonferroni correction for the 9 post-hoc correlations performed (subscale scores in SIPS positive and negative domains, excluding $\mathrm{N} 5 ; p \leq 0.005$ ), only the P2 and P5 correlations survived.

Based on this result, we performed additional correlations between the ISFG and cognitive domains known to be associated with early psychotic symptoms, namely attention and executive functions domains (total of 4 correlations, Bonferroni correction at $p \leq 0.0125$ ). We found a significant correlation with the CPT_Hits t-score $(r=0.49$, $p=0.011$ ).

\section{Discussion}

The three objectives of this study were: 1 ) to identify the functional resting state networks in a group of adolescents affected by 22 q11.2 deletion syndrome (22q11DS); 2) to characterize potential dysconnectivity within these networks; and 3) to examine to which extent dysconnectivity in 22q11DS RSNs related to early manifestations of psychotic symptoms and neuropsychological impairments. Using ICA, we found evidence for nine independent components: a default-mode network (DMN), a self-referential network (SRN), a sensorimotor network (SMN), an auditory network (AN), a median low level (LLVisN) and a lateral high level (HLVisN) visual processing networks, a visuo-spatial processing network (VSPN), a left-lateralized language processing network (LFPN), and a right-lateralized attention network (RAN). Group differences within these networks revealed patterns of dysconnectivity in the 22q11DS group affecting the DMN, the VSPN, the SMN and the 


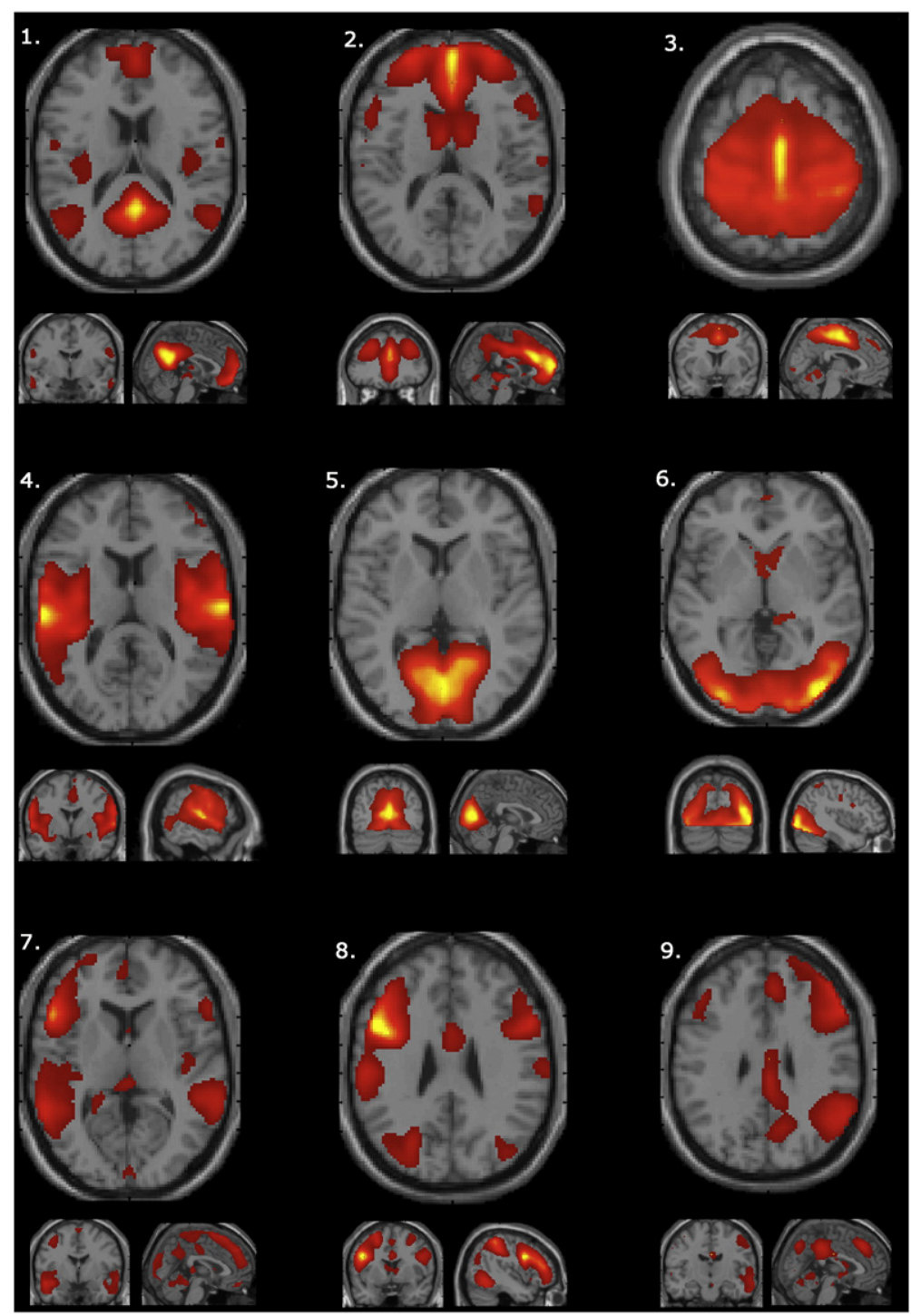

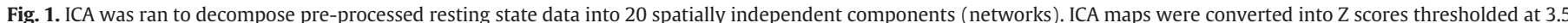

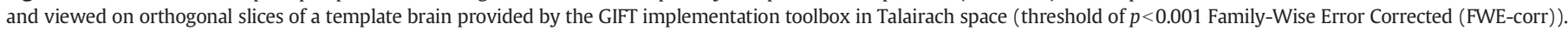

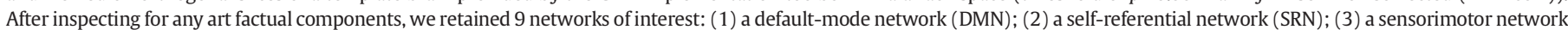

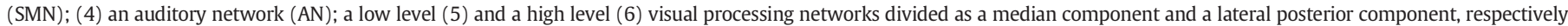
(LLVisN and HLVisN); (7) a visuo-spatial processing network (VSPN); (8) a left-lateralized language processing network (LFPN); (9) a right-lateralized attention network (RAN).

HLVisN. Finally, correlational analyses performed between psychotic symptom and neuropsychological ratings in the 22q11DS group highlighted two areas of significant associations within the left superior frontal gyrus (ISFG) region of the DMN. The discussion will address the issue of dysconnectivity in the resting brain of adolescents with 22q11DS, and discuss how findings regarding the early prodromal signs and neuropsychological characteristics inform the neurodevelopmental account of psychosis in 22q11DS.

Comparing the resting brain's functional connectivity patterns between controls and 22q11DS revealed significant differences in networks known to sustain sensitive cognitive functions in the deletion group. Starting with the visuo-spatial processing network (VSPN), increased activation in the medial parietal lobe is indicative of atypical connectivity involving the left precuneus and posterior cingulate regions. In light of previous structural MRI findings reporting that poor arithmetic abilities in 22q11DS correlate with reduced fractional anisotropy values, particularly on the left parietal tracts (Barnea-Goraly et al., 2003, 2005), the increased activity we observed in the VSPN may be interpreted as the signature of functional dysconnectivity in the dorsal "where" stream, often hypothesized to underlie the characteristic visuo-spatial dysfunctions in children with 22q11DS (LajinessO'Neill et al., 2005; Van Aken et al., 2009). Consistently, parietal lobe gray matter volumes are often found to be reduced in children with 22q11DS (Eliez et al., 2000; Kates et al., 2001), and a recent report suggests that gyrification in the precuneus/PCC regions is more affected in children with 22q11DS as a result of congenital heart defects associated with this deletion syndrome (Schaer et al., 2009b). Observing parietal resting-state functional dysconnectivity for the first time provides the missing link between previously reported structural alterations of parietal gray and white matter (Gothelf et al., 2008) and visuo-spatial/ arithmetic impairments in 22q11DS (Bearden et al., 2001; De Smedt et al., 2007b; Simon et al., 2008). Most probably, this altered cerebral development is sustained by cerebral structure dysmaturation, functional dysconnectivity and their interactions with developing skills in the visuo-spatial domain (Simon et al., 2005, 2008).

Another network showing superior activation peaks in the 22q11DS group is the SMN, around the pre and post-central gyri. Atypical connectivity findings in this network provides converging evidence with 
a

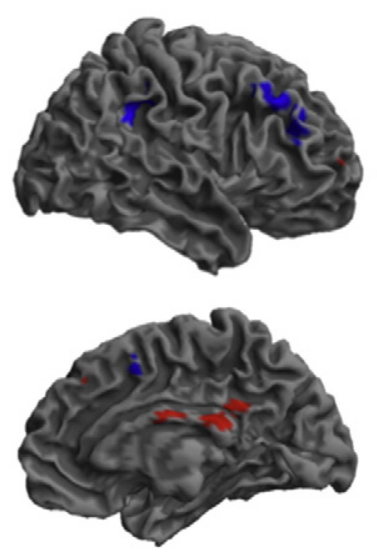

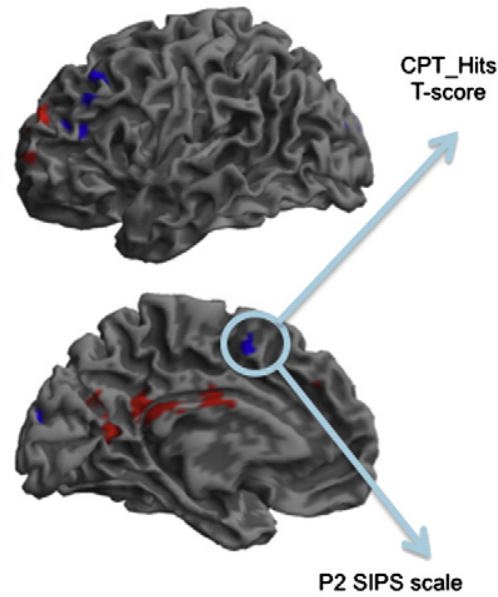

Suspiciousness/

Persecutory Ideas (0-6) b

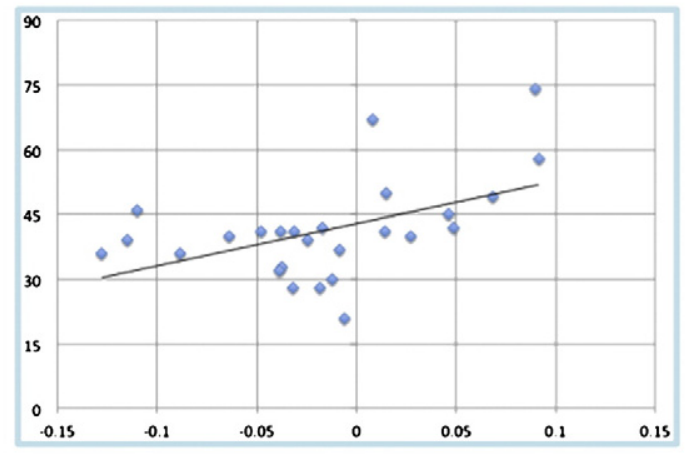

Left SFG eigen value within Default-Mode Network

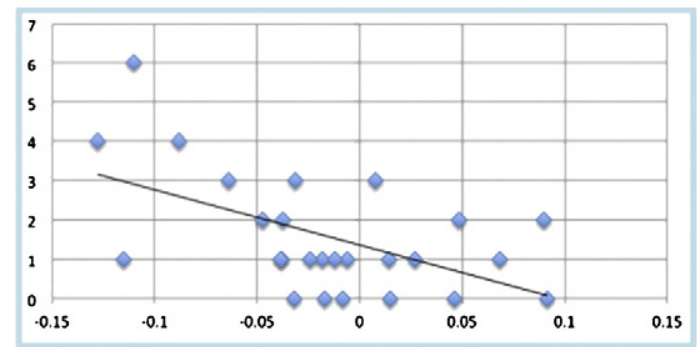

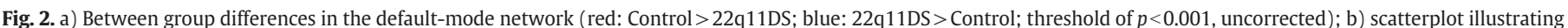

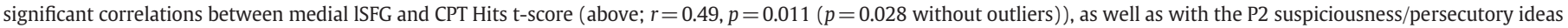
subscale (below; $r=-0.54, p=0.004$ ( $p=0.002$ without outliers)).

several behavioral studies reporting motor deficits (Gerdes et al., 1999), as well as inefficient kinematic movement and motor control strategies (Van Aken et al., 2010a, 2010b), not unlike those found in schizophrenia (Chan and Gottesman, 2008). Along with altered connectivity in the SMN and the "where" dorsal stream, hypo-activation within the high level visual network (HLVisN) is consistent with impairments to the "what" ventral stream in 22q11DS. The HLVisN is thought to sustain cognitive skills such as face processing, orthography and covert reading (Laird et al., 2011), all of which represent important cognitive challenges in 22q11DS (Andersson et al., 2008; Van Aken et al., 2009; Debbané et al., 2010). Together, these results provide the preliminary evidence sustaining the hypothesis associating functional dysconnectivity in the resting brain of adolescents with 22q11DS to relevant cognitive impairments they often experience during development,

Table 2

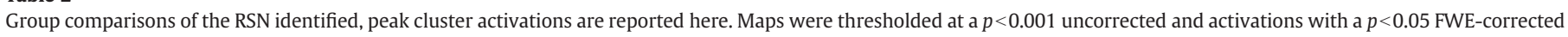
were retained.

\begin{tabular}{|c|c|c|c|c|c|}
\hline Anatomical label & Hem & $\mathrm{BA}$ & MNI (x, y, z) & Peak T-value & $p$ (FWE-corr) cluster \\
\hline \multicolumn{6}{|c|}{ High level visual processing network } \\
\hline \multicolumn{6}{|c|}{ Controls vs. VCFS } \\
\hline Superior parietal lobule & $\mathrm{R}$ & 7 & $21-6755$ & 5.36 & 0.001 \\
\hline Precuneus & $\mathrm{L}$ & 7 & $-21-6452$ & 4.16 & 0.008 \\
\hline \multicolumn{6}{|l|}{ Sensorimotor network } \\
\hline \multicolumn{6}{|l|}{ VCFS vs. controls } \\
\hline Postcentral gyrus & $\mathrm{L}$ & 3 & $-30-3752$ & 4.87 & 0.043 \\
\hline Precentral gyrus & $\mathrm{R}$ & 4 & $30-2864$ & 4.74 & 0.009 \\
\hline \multicolumn{6}{|c|}{ Visuo-spatial processing network } \\
\hline \multicolumn{6}{|c|}{ VCFS vs. controls } \\
\hline Precuneus & $\mathrm{L}$ & 31 & $-18-5834$ & 4.95 & 0.001 \\
\hline \multicolumn{6}{|l|}{ Default mode network } \\
\hline \multicolumn{6}{|l|}{ Controls vs. VCFS } \\
\hline Medial frontal gyrus & $\mathrm{L}$ & 9 & 03534 & 4.94 & $0.036^{\mathrm{a}}$ \\
\hline Cingulate gyrus & $\mathrm{R}$ & 31 & $12-4628$ & 4.91 & $0.001^{\mathrm{a}}$ \\
\hline Superior frontal gyrus & $\mathrm{L}$ & 10 & -245328 & 4.67 & 0.007 \\
\hline Precuneus & $\mathrm{L}$ & 31 & $-6-6728$ & 4.49 & $0.001^{\mathrm{a}}$ \\
\hline \multicolumn{6}{|l|}{ VCFS vs. controls } \\
\hline Middle frontal gyrus & $\mathrm{R}$ & 9 & 392937 & 4.99 & 0.001 \\
\hline Inferior parietal lobule & $\mathrm{R}$ & 40 & $57-3728$ & 4.81 & 0.009 \\
\hline Middle frontal gyrus & $\mathrm{L}$ & 8 & -362946 & 4.76 & 0.015 \\
\hline Superior frontal gyrus & $\mathrm{L}$ & 6 & 0255 & 4.41 & $0.01^{\mathrm{a}}$ \\
\hline Cuneus & $\mathrm{L}$ & 19 & $-9-9125$ & 4.35 & $0.04^{\mathrm{a}}$ \\
\hline
\end{tabular}

\footnotetext{
a Cortical midline regions in the DMN selected for regression model; peak activation clusters, ROIs were extracted from these coordinates.
} 
specifically those touching upon the dorsal "where" stream, the sensorimotor network and the ventral visual "what" stream.

While the atypical connectivity patterns within the VSPN, SMN and HLVisN suggest a number of relationships with typical cognitive and motor impairments in 22q11DS, the pattern found in the default-mode network (DMN) further relates to early symptoms of psychosis. In the 22q11DS group, increased activation of the posterior medial ISFG was negatively associated to prodromal expression of emotion, suspicious/persecutory ideas and disorganized communication, and positively associated to impulse control and attention. These results are consistent with a meta-analysis covering fMRI studies using emotion processing and executive function paradigms in high-risk for psychosis samples (Fusar-Poli, 2012). Compared to controls in such tasks, this meta-analysis found hypo-activation in SFG, as in other prefrontal areas, to be characteristic of high-risk for psychosis during adolescence. Consistently, our current findings suggest that posterior medial ISFG peak activation in the 22q11DS group's DMN represents a compensatory mechanism associated with lower delusional beliefs and more appropriate emotional expression, as well as better regulation of impulsivity and attention.

The investigation of functional connectivity in 22q11DS raises a number of questions. First, as recently underlined (Van Dijk et al., 2012), increased precaution regarding head movement during resting state acquisition in pediatric samples significantly increases the integrity of results. Second, the relationship between functional connectivity and IQ represents a complex, multi-determined developmental issue. A recent study examining this relationship reported that measures of network efficiency in neurotypicals, especially in frontal and parietal lobes, seem to account for some variation in intellectual performance (van den Heuvel et al., 2009). These findings are relevant to 22q11DS, who typically show altered cerebral structure, namely in the parietal lobe. Integrating both structural and functional connectivity measures in future studies may tackle such central issues concerning cerebral connectivity.

\section{Role of funding source}

This research was supported by research grants from the Swiss National Science Foundation grant to Stephan Eliez (PP00B-102864), Martin Debbané (100014135311/1), and Dimitri Van de Ville (PP00P2-123438), and in part by the Center for Biomedical Imaging (CIBM) of the Geneva and Lausanne Universities, and EPFL. These funding bodies had no role in the analysis and interpretation of data, in the writing of the manuscript, or in the decision concering submission for publication.

\section{Contributors}

Authors M.D. and S.E. designed the study and wrote the protocol. Authors M.L., A.L. and M.S. managed the data collection and analyses. Authors M.L. and D.V.d.V. undertook the statistical analysis, and author M.D. wrote the first draft of the manuscript. All authors contributed to and have approved the final manuscript.

\section{Conflict of interest}

None of the authors has any conflict of interest to report.

\section{Acknowledgments}

We wish to thank all the participants and their families who kindly volunteered for this study. We extend our special thanks to Sarah Menghetti and Eleonora Rizzi for help in data management.

\section{References}

Alexander-Bloch, A.F., Gogtay, N., Meunier, D., Birn, R., Clasen, L., Lalonde, F., Lenroot, R., Giedd, J., Bullmore, E.T., 2010. Disrupted modularity and local connectivity of brain functional networks in childhood-onset schizophrenia. Front. Syst. Neurosci. 4, 147.

Alexander-Bloch, A.F., Vertes, P.E., Stidd, R., Lalonde, F., Clasen, L., Rapoport, J., Giedd, J., Bullmore, E.T., Gogtay, N., 2012. The anatomical distance of functional connections predicts brain network topology in health and schizophrenia. Cereb. Cortex.

Andersson, F., Glaser, B., Spiridon, M., Debbane, M., Vuilleumier, P., Eliez, S., 2008. Impaired activation of face processing networks revealed by functional magnetic resonance imaging in 22q11.2 deletion syndrome. Biol. Psychiatry 43, 49-57.

Assaf, M., Jagannathan, K., Calhoun, V.D., Miller, L., Stevens, M.C., Sahl, R., O'Boyle, J.G., Schultz, R.T., Pearlson, G.D., 2010. Abnormal functional connectivity of default mode sub-networks in autism spectrum disorder patients. Neurolmage 53 (1), 247-256.
Barnea-Goraly, N., Menon, V., Krasnow, B., Ko, A., Reiss, A., Eliez, S., 2003. Investigation of white matter structure in velocardiofacial syndrome: a diffusion tensor imaging study. Am. J. Psychiatry 160 (10), 1863-1869.

Barnea-Goraly, N., Eliez, S., Menon, V., Bammer, R., Reiss, A.L., 2005. Arithmetic ability and parietal alterations: a diffusion tensor imaging study in velocardiofacial syndrome. Brain Res. Cogn. Brain Res. 25 (3), 735-740.

Bearden, C.E., Woodin, M.F., Wang, P.P., Moss, E., McDonald-McGinn, D., Zackai, E., Emannuel, B., Cannon, T.D., 2001. The neurocognitive phenotype of the 22q11.2 deletion syndrome: selective deficit in visual-spatial memory. J. Clin. Exp. Neuropsychol. 23 (4), 447-464.

Broyd, S.J., Demanuele, C., Debener, S., Helps, S.K., James, C.J., Sonuga-Barke, E.J., 2009 Default-mode brain dysfunction in mental disorders: a systematic review. Neurosci. Biobehav. Rev. 33 (3), 279-296.

Chan, R.C., Gottesman, I.I., 2008. Neurological soft signs as candidate endophenotypes for schizophrenia: a shooting star or a Northern star? Neurosci. Biobehav. Rev. 32 (5), 957-971.

De Smedt, B., Devriendt, K., Fryns, J.P., Vogels, A., Gewillig, M., Swillen, A., 2007a. Intellectual abilities in a large sample of children with velo-cardio-facial syndrome: an update. J. Intellect. Disabil. Res. 51 (Pt 9), 666-670.

De Smedt, B., Swillen, A., Devriendt, K., Fryns, J.P., Verschaffel, L., Ghesquiere, P., 2007b. Mathematical disabilities in children with velo-cardio-facial syndrome. Neuropsychologia 45 (5), 885-895.

Debbané, M., Van der Linden, M., Glaser, B., Eliez, S., 2008. Source monitoring for actions in adolescents with 22q11.2 deletion syndrome (22q11DS). Psychol. Med. 38 (6), 811-820.

Debbané, M., Van der Linden, M., Glaser, B., Eliez, S., 2010. Monitoring of self-generated speech in adolescents with 22q11.2 deletion syndrome. Br. J. Clin. Psychol. 49 (Pt 3), 373-386.

Eisenberg, D.P., Jabbi, M., Berman, K.F., 2010. Bridging the gene-behavior divide through neuroimaging deletion syndromes: velocardiofacial (22q11.2 deletion) and Williams (7q11.23 deletion) syndromes. NeuroImage 53 (3), 857-869.

Eliez, S., Schmitt, J.E., White, C.D., Reiss, A.L., 2000. Children and adolescents with velocardiofacial syndrome: a volumetric MRI study. Am. J. Psychiatry 157 (3), 409-415.

Fair, D.A., Posner, J., Nagel, B.J., Bathula, D., Dias, T.G., Mills, K.L., Blythe, M.S., Giwa, A. Schmitt, C.F., Nigg, J.T., 2010. Atypical default network connectivity in youth with attention-deficit/hyperactivity disorder. Biol. Psychiatry 68 (12), 1084-1091.

Friston, K.J., 1998. The disconnection hypothesis. Schizophr. Res. 30 (2), 115-125.

Friston, K.J., Frith, C.D., 1995. Schizophrenia: a disconnection syndrome? Clin. Neurosci. 3 (2), 89-97.

Fusar-Poli, P., 2012. Voxel-wise meta-analysis of fMRI studies in patients at clinical high risk for psychosis. J. Psychiatry Neurosci. 37 (2), 106-112.

Gerdes, M., Solot, C., Wang, P.P., Moss, E., LaRossa, D., Randall, P., Goldmuntz, E., Clark III, B.J., Driscoll, D.A., Jawad, A., Emanuel, B.S., McDonald-McGinn, D.M., Batshaw, M.L., Zackai, E.H., 1999. Cognitive and behavior profile of preschool children with chromosome 22q11.2 deletion. Am. J. Med. Genet. 85 (2), 127-133.

Gothelf, D., Schaer, M., Eliez, S., 2008. Genes, brain development and psychiatric phenotypes in velo-cardio-facial syndrome. Dev. Disabil. Res. Rev. 14 (1), 59-68.

Gothelf, D., Hoeft, F., Ueno, T., Sugiura, L., Lee, A.D., Thompson, P., Reiss, A.L., 2011. Developmental changes in multivariate neuroanatomical patterns that predict risk for psychosis in 22q11.2 deletion syndrome. J. Psychiatr. Res. 45 (3), 322-331.

Green, T., Gothelf, D., Glaser, B., Debbane, M., Frisch, A., Kotler, M., Weizman, A., Eliez, S., 2009. Psychiatric disorders and intellectual functioning throughout development in velocardiofacial (22q11.2 deletion) syndrome. J. Am. Acad. Child Adolesc. Psychiatry 48 (11), 1060-1068.

Greicius, M., 2008. Resting-state functional connectivity in neuropsychiatric disorders Curr. Opin. Neurol. 21 (4), 424-430.

Hoffman, R.E., McGlashan, T.H., 2001. Neural network models of schizophrenia. Neuroscientist 7 (5), 441-454.

Kates, W.R., Burnette, C.P., Jabs, E.W., Rutberg, J., Murphy, A.M., Grados, M., Geraghty, M. Kaufmann, W.E., Pearlson, G.D., 2001. Regional cortical white matter reductions in velocardiofacial syndrome: a volumetric MRI analysis. Biol. Psychiatry 49 (8), 677-684

Lagioia, A.L., Van de Ville, D., Debbané, M., Lazeyras, F., Eliez, S., 2010. Adolescent resting state networks and their associations with schizotypal trait expression. Front Syst. Neurosci. 35 (4), 1-12.

Laird, A.R., Fox, P.M., Eickhoff, S.B., Turner, J.A., Ray, K.L., McKay, D.R., Glahn, D.C., Beckmann, C.F., Smith, S.M., Fox, P.T., 2011. Behavioral interpretations of intrinsic connectivity networks. J. Cogn. Neurosci. 23 (12), 4022-4037.

Lajiness-O'Neill, R.R., Beaulieu, I., Titus, J.B., Asamoah, A., Bigler, E.D., Bawle, E.V., Pollack, R., 2005. Memory and learning in children with 22q11.2 deletion syndrome: evidence for ventral and dorsal stream disruption? Child Neuropsychol. 11 (1), 55-71.

Li, Y.O., Adali, T., Calhoun, V.D., 2007. Estimating the number of independent components for functional magnetic resonance imaging data. Hum. Brain Mapp. 28 (11), 1251-1266.

Lynall, M.E., Bassett, D.S., Kerwin, R., McKenna, P.J., Kitzbichler, M., Muller, U., Bullmore, E., 2010. Functional connectivity and brain networks in schizophrenia. J. Neurosci. 30 (28), 9477-9487.

Mantini, D., Perrucci, M.G., Del Gratta, C., Romani, G.L., Corbetta, M., 2007. Electrophysiological signatures of resting state networks in the human brain. Proc. Natl. Acad. Sci. U. S. A. 104 (32), 13170-13175.

McGlashan, T., 2001. Structured Interview for Prodromal Syndromes (SIPS). New Haven.

Murphy, K.C., Jones, L.A., Owen, M.J., 1999. High rates of schizophrenia in adults with velo-cardio-facial syndrome. Arch. Gen. Psychiatry 56 (10), 940-945.

Oskarsdottir, S., Vujic, M., Fasth, A., 2004. Incidence and prevalence of the 22q11 deletion syndrome: a population-based study in Western Sweden. Arch. Dis. Child. 89 (2), 148-151. 
Raichle, M.E., Snyder, A.Z., 2007. A default mode of brain function: a brief history of an evolving idea. Neurolmage 37 (4), 1083-1090 (discussion 1097-1089).

Rosazza, C., Minati, L., 2011. Resting-state brain networks: literature review and clinical applications. Neurol. Sci. 32 (5), 773-785.

Schaer, M., Debbané, M., Bach Cuadra, M., Ottet, M.C., Glaser, B., Thiran, J.P., Eliez, S. 2009a. Deviant trajectories of cortical maturation in 22q11.2 deletion syndrome (22q11DS): a cross-sectional and longitudinal study. Schizophr. Res. 115 (2-3) 182-190.

Schaer, M., Glaser, B., Cuadra, M.B., Debbane, M., Thiran, J.P., Eliez, S., 2009b. Congenital heart disease affects local gyrification in 22q11.2 deletion syndrome. Dev. Med. Child Neurol. 51 (9), 746-753.

Schneider, M., Van der Linden, M., Glaser, B., Rizzi, E., Dahoun, S.P., Hinard, C., Bartoloni, L., Antonarakis, S.E., Debbane, M., Eliez, S., 2012. Preliminary structure and predictive value of attenuated negative symptoms in 22q11.2 deletion syndrome. Psychiatry Res. 196 (2-3), 277-284.

Shim, G., Oh, J.S., Jung, W.H., Jang, J.H., Choi, C.H., Kim, E., Park, H.Y., Choi, J.S., Jung, M.H. Kwon, J.S., 2010. Altered resting-state connectivity in subjects at ultra-high risk for psychosis: an fMRI study. Behav. Brain Funct. 6, 58.

Simon, T.J., Bearden, C.E., Mc-Ginn, D.M., Zackai, E., 2005. Visuospatial and numerical cognitive deficits in children with chromosome 22q11.2 deletion syndrome. Cortex 41 (2), 145-155.

Simon, T.J., Wu, Z., Avants, B., Zhang, H., Gee, J.C., Stebbins, G.T., 2008. Atypical cortical connectivity and visuospatial cognitive impairments are related in children with chromosome 22q11.2 deletion syndrome. Behav. Brain Funct. 4, 25.
Sorg, C., Manoliu, A., Neufang, S., Myers, N., Peters, H., Schwerthoffer, D., Scherr, M., Muhlau, M., Zimmer, C., Drzezga, A., Forstl, H., Bauml, J., Eichele, T., Wohlschlager, A.M., Riedl, V., in press. Increased intrinsic brain activity in the striatumreflects symptom dimensions in schizophrenia. Schizophr. Bull.

Stevens, M.C., Kiehl, K.A., Pearlson, G., Calhoun, V.D., 2007. Functional neural circuits for mental timekeeping. Hum. Brain Mapp. 28 (5), 394-408.

Stoddard, J., Niendam, T., Hendren, R., Carter, C., Simon, T.J., 2010. Attenuated positive symptoms of psychosis in adolescents with chromosome 22q11.2 deletion syndrome. Schizophr. Res. 118 (1-3), 118-121.

Van Aken, K., Caeyenberghs, K., Smits-Engelsman, B., Swillen, A., 2009. The motor profile of primary school-age children with a 22q11.2 deletion syndrome (22q11.2DS) and an age- and IQ-matched control group. Child Neuropsychol. 15 (6), 532-542.

Van Aken, K., Swillen, A., Beirinckx, M., Janssens, L., Caeyenberghs, K., Smits-Engelsman, B., 2010a. Kinematic movement strategies in primary school children with $22 \mathrm{q} 11.2$ deletion syndrome compared to age- and IQ-matched controls during visuomanual tracking. Res. Dev. Disabil. 31 (3), 768-776.

Van Aken, K., Swillen, A., Beirinckx, M., Janssens, L., Caeyenberghs, K., SmitsEngelsman, B., 2010b. Prospective control abilities during visuo-manual tracking in children with 22q11.2 deletion syndrome compared to age- and IQ-matched controls. Res. Dev. Disabil. 31 (3), 634-641.

van den Heuvel, M.P., Stam, C.J., Kahn, R.S., Hulshoff Pol, H.E., 2009. Efficiency of functional brain networks and intellectual performance. J. Neurosci. 29 (23), 7619-7624.

Van Dijk, K.R., Sabuncu, M.R., Buckner, R.L., 2012. The influence of head motion on intrinsic functional connectivity MRI. NeuroImage 59 (1), 431-438. 